\title{
Utility-preserving anonymization for health data publishing
}

\author{
Hyukki Lee ${ }^{1}$, Soohyung Kim², Jong Wook Kim³ and Yon Dohn Chung ${ }^{1 *}$ (D)
}

\begin{abstract}
Background: Publishing raw electronic health records (EHRs) may be considered as a breach of the privacy of individuals because they usually contain sensitive information. A common practice for the privacy-preserving data publishing is to anonymize the data before publishing, and thus satisfy privacy models such as k-anonymity. Among various anonymization techniques, generalization is the most commonly used in medical/health data processing. Generalization inevitably causes information loss, and thus, various methods have been proposed to reduce information loss. However, existing generalization-based data anonymization methods cannot avoid excessive information loss and preserve data utility.
\end{abstract}

Methods: We propose a utility-preserving anonymization for privacy preserving data publishing (PPDP). To preserve data utility, the proposed method comprises three parts: (1) utility-preserving model, (2) counterfeit record insertion, (3) catalog of the counterfeit records. We also propose an anonymization algorithm using the proposed method. Our anonymization algorithm applies full-domain generalization algorithm. We evaluate our method in comparison with existence method on two aspects, information loss measured through various quality metrics and error rate of analysis result.

Results: With all different types of quality metrics, our proposed method show the lower information loss than the existing method. In the real-world EHRs analysis, analysis results show small portion of error between the anonymized data through the proposed method and original data.

Conclusions: We propose a new utility-preserving anonymization method and an anonymization algorithm using the proposed method. Through experiments on various datasets, we show that the utility of EHRs anonymized by the proposed method is significantly better than those anonymized by previous approaches.

Keywords: Medical privacy, Data anonymization, Utility-preserving data publishing, K-anonymity

\section{Background Motivation}

In recent years, various health and medical institutions have collected a large amount of medical data, called Electronic Health Records (EHRs). These data are valuable resources that can be used for the prevention of disease, medical decision making, and many other areas of healthcare. Furthermore, various medical data besides EHRs are also widely used in the health domain [1,2]. Accordingly, data owners have attempted to use the data gathered to

\footnotetext{
*Correspondence: ydchung@korea.ac.kr

'Department of Computer Science and Engineering, Korea University, 145

Anam-ro, Seongbuk-gu, 02841, Seoul, Republic of Korea

Full list of author information is available at the end of the article
}

make profits through publishing or outsourcing of the data to research organizations.

However, the EHR data usually contain sensitive information such as diagnosis and medication. If data subjects pertaining to sensitive information are disclosed to others, privacy can be breached. For this reason, many countries protect individuals' privacy in data publishing by laws. These laws allow the publication of only privacypreserved data. For example, the US Health Insurance Portability and Accountability Act (HIPAA) privacy rule grants the publication of medical information for public purposes without a patient's consent, if the privacy is preserved adequately. In European Union, privacy regulations are more strict. If data privacy is breached, the data have 
to be erased [3]. Data anonymization can preserve privacy by eliminating identifiability from the dataset, i.e., the link between sensitive information and people. However, removing Personally Identifiable Information (PII) is not sufficient for eliminating identifiability. A combination of characteristic information (e.g., sex, zipcode, and age), called quasi-identifiers, can play the role of an identifier [4]. To prevent the breach of privacy by quasi-identifiers, $k$-anonymity was proposed [4]. $k$-anonymity means each record contained in a released dataset cannot be distinguished from at least $k-1$ other individuals. $k$-anonymity is used as the privacy criteria in real applications such as the 'Family Educational Rights and Privacy Act' (FERPA) [5] of US and the 'Guidelines for De-identification of Personal Data' of South Korea [6].

A typical method to achieve $k$-anonymity is generalization. Generalization involves transforming values of quasi-identifiers into more general values to make a person indiscernible from several people. For example, suppose that we release a 4-anonymous dataset for the raw EHR dataset in Table 1, in which name is a direct identifier, age, sex, and zipcode are quasi-identifiers, and disease is a sensitive attribute. The identifier is removed, and the quasi-identifiers are generalized to anonymize the data; the sensitive information should not be removed or modified because it is a critical attribute for analysis. The anonymized result is shown in Table 2. Each record is generalized to an indistinguishable group, called the equivalent class, <[35-66], *, [22071-55324] >. As a result of generalization, each record is related to at least three identical records, and thus, the dataset in Table 2 satisfies the 4-anonymity.

Note that anonymization achieved with generalizationonly approaches inevitably distort the records. Therefore, over-generalization negatively affects the analysis of the anonymized dataset. For example, when performing the age-period-cohort analysis using Table 2, as the age is over-generalized, it is difficult to obtain meaningful results. In addition, it is important to prevent over-generalization, as the "doctor in the loop" paradigm increases, which the expert knowledge of the doctor is

Table 1 Original EHR data

\begin{tabular}{lllll}
\hline Name & Age & Sex & Zipcode & Disease \\
\hline Mary & 37 & F & 22071 & Pneumonia \\
Alice & 35 & F & 22098 & Diabetes \\
Betsy & 36 & F & 23061 & Anemia \\
David & 61 & M & 55107 & Pneumonia \\
Tom & 63 & M & 55099 & Diabetes \\
James & 66 & M & 55324 & Diabetes \\
Eric & 62 & M & 55229 & Pneumonia \\
\hline
\end{tabular}

Table 2 4-anonymous version of Table 1

\begin{tabular}{llll}
\hline Age & Sex & Zipcode & Disease \\
\hline$[35-66]$ & $*$ & {$[22071-55324]$} & Pneumonia \\
{$[35-66]$} & $*$ & {$[22071-55324]$} & Diabetes \\
{$[35-66]$} & $*$ & {$[22071-55324]$} & Anemia \\
{$[35-66]$} & $*$ & {$[22071-55324]$} & Pneumonia \\
{$[35-66]$} & $*$ & {$[22071-55324]$} & Diabetes \\
{$[35-66]$} & $*$ & {$[22071-55324]$} & Diabetes \\
{$[35-66]$} & $*$ & {$[22071-55324]$} & Pneumonia \\
\hline
\end{tabular}

incorporated into "intelligent" systems (e.g., using interactive machine learning) and enriched with additional information and expert know-how [7].

However, anonymization with generalization-only approaches cannot prevent over-generalization, because there is no other way to organize an equivalent class that consists of dissimilar records for achieving $k$-anonymity. To reduce over-generalization, several approaches were proposed [8-10]. Nevertheless, these methods still cannot avoid over-generalization for all records. Therefore, we propose utility-preserving anonymization to achieve $k$-anonymity with high utility. In this method, we propose a utility-preserving model to guarantee the prevention of over-generalization. Furthermore, we propose an anonymization method that satisfies the proposed model and preserves privacy as well.

\section{Generalization, suppression, and relocation}

In order to generalize the records, numerical attribute values are transformed into range values, and categorical attribute values are transformed into superordinate values. Taxonomy trees are usually employed to describe hierarchies of categorical attributes. Figure 1 shows the taxonomy tree of Age attribute. The degree of generalization is quantified as a number between 0 (i.e., minimum generalization) and 1 (i.e., maximum generalization). The degrees of generalization for a numerical attribute $a_{\text {num }}$ and a categorical attribute $a_{c a t}$ are computed as follows.

$$
\begin{aligned}
& D_{A}\left(a_{\text {num }}\right)=\frac{U_{q}-L_{q}}{U-L} \\
& D_{A}\left(a_{c a t}\right)=\frac{\left|M_{p}\right|-1}{|M|-1}
\end{aligned}
$$

$U_{q}$ and $L_{q}$ denote the upper bound and lower bound of the generalized interval, respectively. $U$ and $L$ denote the maximum and minimum value of the whole domain, respectively. $M$ denotes the set of leaf nodes in the taxonomy tree and $M_{p}$ denotes the set of leaf nodes of the subtree rooted by the generalized node. The degree of generalization for a record is defined as the average value of each quasi-identifier attribute's generalization degree. 


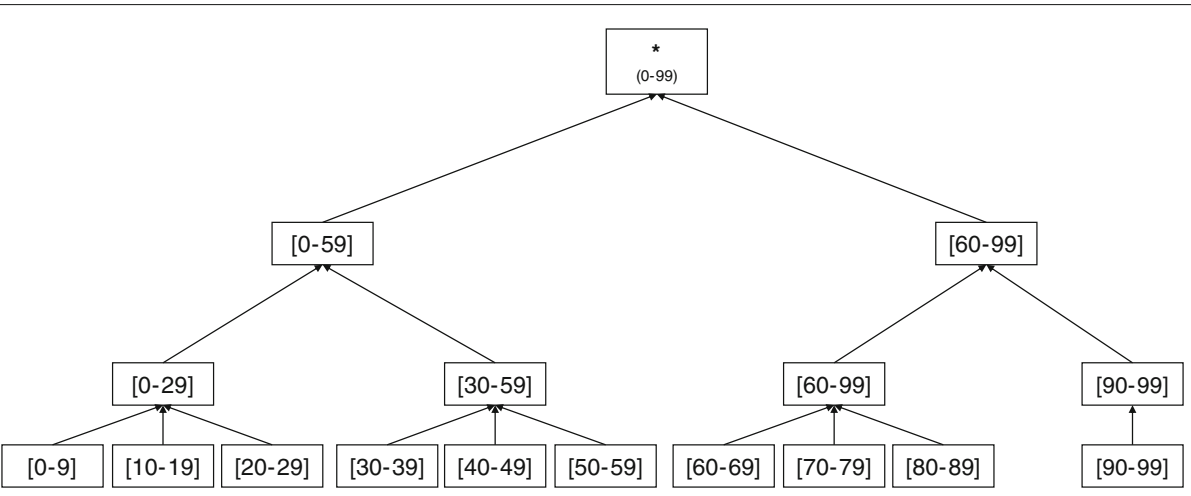

Fig. 1 Taxonomy tree of Age attribute

The degree of generalization for the record $r$ is

$$
D(r)=\frac{1}{n} \sum_{i=1}^{n} D_{A}\left(a_{i}\right)
$$

where $n$ is the number of attributes. For instance, in the 4-anonymous Table 2, Age is a numerical attribute and its domain is [0-99]. Generalization degree for Age of the first record is $(66-35) /(99-0)=0.31$. The other attributes' generalization degrees are $(2-1) /(2-1)=1$ and $(33254-1) /(100000-1)=0.33$, respectively. Therefore, the generalization degree of the first record is $(0.31+1+0.33) / 3=0.55$.

There are two types of anonymization methods that are used in conjunction with generalization for reducing over-generalization: suppression and relocation $[8,9]$. Suppression involves the removal of outliers, and relocation involves the changing of the quasi-identifiers of outliers. The presence of outliers is one of the main causes of over-generalization. This is because the outliers are distant from other records, as well as the number of them is not sufficient that they can organize equivalent classes by themselves. In these methods, the values of outliers are removed or changed. Therefore, they can avoid organizing an equivalent class that collects the outlier with other records, and can less generalize a majority of records. Table 3 is obtained from Table 1 by suppressing the first

Table 3 4-anonymous version of Table 1 with suppression

\begin{tabular}{llll}
\hline Age & Sex & Zipcode & Disease \\
\hline$*$ & $*$ & $*$ & $*$ \\
$*$ & $*$ & $*$ & $*$ \\
$*$ & $*$ & $*$ & $*$ \\
{$[61-66]$} & $M$ & {$[55099-55324]$} & Pneumonia \\
{$[61-66]$} & $M$ & {$[55099-55324]$} & Diabetes \\
{$[61-66]$} & $M$ & {$[55099-55324]$} & Diabetes \\
{$[61-66]$} & $M$ & {$[55099-55324]$} & Pneumonia \\
\hline
\end{tabular}

three records. On the other hands, Table 4 is acquired by relocating the first three records in Table 1. Quasiidentifiers of the first three records are relocated to the quasi-identifiers of the other records. By the suppression and relocation of outlier records, the remaining records are not over-generalized.

\section{Data utility}

The main objective of $k$-anonymization is privacy protection; however, it is also important that the anonymized dataset should be as useful as possible. There are various $k$-anonymization of a given dataset, but one having the highest utility is desirable. In PPDP, a data owner does not know how the published data will be analyzed by recipients, thus the data utility is measured by the quality of the anonymized dataset. We mainly focus on information loss and data truthfulness for assessing data utility, because these can cover the entire quality of the anonymized dataset in the proposed method (the details will be described later). Information loss refers to the amount of loss caused by generalization. Data truthfulness implies that each anonymized record corresponds to a single original record [11]. Relocated records cannot correspond to original records; thus, they are untruthful. In privacy-preserving data publishing, it is important that a published dataset is truthful. If a published dataset is not truthful, it is difficult to use the results of the data analysis,
Table 4 4-anonymous version of Table 1 with relocation

\begin{tabular}{llll}
\hline Age & Sex & Zipcode & Disease \\
\hline$[61-66]$ & $M$ & {$[55099-55324]$} & Pneumonia \\
{$[61-66]$} & $M$ & {$[55099-55324]$} & Diabetes \\
{$[61-66]$} & $M$ & {$[55099-55324]$} & Anemia \\
{$[61-66]$} & $M$ & {$[55099-55324]$} & Pneumonia \\
{$[61-66]$} & $M$ & {$[55099-55324]$} & Diabetes \\
{$[61-66]$} & $M$ & {$[55099-55324]$} & Diabetes \\
{$[61-66]$} & $M$ & {$[55099-55324]$} & Pneumonia \\
\hline
\end{tabular}


because false-positive and false-negative results may be obtained. For example, in Table 1, there is a female patient with Anemia in her 30s. However, in Table 4, there is no female Anemia patient, and in addition, it contains a male Anemia patient in his 60s that does not exist in Table 1.

To quantify data utility, various quality metrics are proposed, such as classification metric discernibility metric (DM) [12], loss metric (LM) [13], and reconstruction error (RCE) [14]. DM measures the cardinality of the equivalent class. DM considers only the number of records in the equivalent class; thus, DM does not capture information loss caused by generalization. LM can measure both the cardinality of the equivalent class and information loss. Although LM is more accurate when measuring information loss, it does not consider the data truthfulness. RCE measures the similarity between the original record and the anonymous record. This metric can reflect both information loss and data truthfulness.

\section{Limitations of the previous methods}

While previous methods such as suppression and relocation could reduce information loss, they have some shortcomings that we will here discuss. First, the number of records that can be relocated or suppressed is limited. Suppression and relocation harm data truthfulness. Therefore, data suppression and relocation are only performed on negligible amounts of records for preserving data truthfulness. If the number of outliers exceeds the limitation, over-generalization cannot be prevented, which leads to unacceptable information loss. For example, in Table 4, there are three relocated records. However, the relocation method cannot be applied, if the number of relocatable records is limited to less than $40 \%$ of the total number of records. Second, the quality metric they employed does not measure data truthfulness [9]. In hybrid $k$-anonymity, an LM is used. LM measures information loss caused only by generalization, and not by relocation [13]. For this reason, data utility of anonymized data can be severely decreased despite low LM.

\section{Methods}

\section{Basic concepts}

In this section, we introduce the basic concepts behind the proposed anonymization method. The three main goals of the proposed method are as follows: the anonymized dataset should remain (1) useful, (2) privacy-preserving, and (3) reliable. In other words, the anonymized dataset should not be over-generalized, and it should satisfy the privacy model ( $k$-anonymity in this paper) and be truthful. To meet these goals, the proposed method comprises three parts: (1) The first part is to restrict the generalization by using a utility-preserving model, called $h$ ceiling, which implies that the degree of generalization is limited to $h$ (Subsection h-ceiling). (2) The second part is to generalize counterfeit records. Both the $k$ anonymity and $h$-ceiling are satisfied by inserting counterfeit records (Subsection Insertion of counterfeit records). (3) The third part is to publish a catalog of the counterfeit records that were inserted in the second part to improve data truthfulness (Subsection Catalog of counterfeit records). The catalog consists of sensitive information of counterfeit records and their number in each group of equivalent class. In addition, we describe a quality metric from the results of the proposed method and propose the implementation of an anonymization algorithm using the proposed method (Subsection Implementation of anonymization algorithm).

\section{h-ceiling}

To prevent over-generalization, we limit the generalization degree to $h$, which is the criterion for overgeneralization. We now formally define the concepts of h-ceiling.

Definition 1 (h-ceiling) Let OT be an original table and AT be an anonymized table of OT. AT satisfies h-ceiling if and only if $D(r) \leq h$.

For example, Table 5 satisfies 0.02 -ceiling because $D\left(r_{1}\right), \ldots, D\left(r_{4}\right)=(2 / 99+0 / 1+990 / 99999) / 3=0.01$ and $D\left(r_{5}\right), \ldots, D\left(r_{8}\right)=(6 / 99+0 / 1+222 / 99999) / 3=0.02$.

\section{Insertion of counterfeit records}

We describe the insertion of counterfeit records for achieving $h$-ceiling and $k$-anonymity. The counterfeit records are inserted into equivalent classes that satisfy $h$ ceiling but not $k$-anonymity. The counterfeit records have the same quasi-identifiers as the records of the equivalent class, while the sensitive information is randomly selected within the domain of the sensitive attribute. For example, Table 6 cannot satisfy $k$-anonymity, because there are only three records in class 1 . Therefore, the counterfeit record is inserted into class 1 to satisfy 4-anonymity as shown in Table 5. A counterfeit record is defined as follows.

Table 5 0.02-ceiled and 4-anonymous version of Table 1 with insertion

\begin{tabular}{lllll}
\hline ClassID & Age & Sex & Zipcode & Disease \\
\hline 1 & {$[35-37]$} & F & {$[22071-23061]$} & Pneumonia \\
1 & {$[35-37]$} & $F$ & {$[22071-23061]$} & Diabetes \\
1 & {$[35-37]$} & $F$ & {$[22071-23061]$} & Anemia \\
1 & {$[35-37]$} & F & {$[22071-23061]$} & Diabetes \\
2 & {$[61-66]$} & M & {$[55099-55324]$} & Pneumonia \\
2 & {$[61-66]$} & M & {$[55099-55324]$} & Diabetes \\
2 & {$[61-66]$} & M & {$[55099-55324]$} & Diabetes \\
2 & {$[61-66]$} & M & {$[55099-55324]$} & Pneumonia \\
\hline
\end{tabular}


Table 6 0.02-ceiled version of Table 1

\begin{tabular}{lllll}
\hline ClassID & Age & Sex & Zipcode & Disease \\
\hline 1 & {$[35-37]$} & F & {$[22071-23061]$} & Pneumonia \\
1 & {$[35-37]$} & F & {$[22071-23061]$} & Diabetes \\
1 & {$[35-37]$} & F & {$[22071-23061]$} & Anemia \\
2 & {$[61-66]$} & $M$ & {$[55099-55324]$} & Pneumonia \\
2 & {$[61-66]$} & $M$ & {$[55099-55324]$} & Diabetes \\
2 & {$[61-66]$} & $M$ & {$[55099-55324]$} & Diabetes \\
2 & {$[61-66]$} & $M$ & {$[55099-55324]$} & Pneumonia \\
\hline
\end{tabular}

Definition 2 (Counterfeit record) We define a record $r \in A T$ is a counterfeit record, if and only if $f^{-1}(r) \notin O T$, where $f()$ be an anonymization function and $f^{-1}()$ be an inverse function of $f()$

For example, Table 5 is an anonymized table that satisfies 0.02 -ceiling and 4-anonymity by adding one counterfeit record, < [35-37], F, [22071-23061], Diabetes $>$ (fourth record).

\section{Catalog of counterfeit records}

The definition and schema of the catalog of counterfeit records are as follows.

Definition 3 (Catalog for counterfeit records) Let $E_{t}(t=1, \ldots, l)$ be an equivalent class in AT. A group of equivalent classes $G_{i}(i=1, \ldots, m)$ consists of several equivalent classes in $E_{t}$. The catalog has schema

\section{Catalog(ClassID list, Sensitive value, Count)}

where ClassIdList is a list of equivalent class ids in same $G_{i}$.

The ID list should be organized considering for privacy during creation of the catalog; else, privacy can be breached by the catalog. For example, some adversaries obtain Table 5, and Table 7 and they try to extract personal information. They can remove a record that has Diabetes in class 1 . Then, only three indistinguishable records exist in class 1; hence 4-anonymity is breached. Although an adversary has some information on counterfeit records, the adversary cannot eliminate counterfeit records when the counterfeit records are not identified [15]. The following lemma shows when the records cannot be eliminated.

Table 7 Privacy breached catalog of counterfeit records for Table 5

\begin{tabular}{lll}
\hline IDList & Disease & Count \\
\hline 1 & Diabetes & 1 \\
\hline
\end{tabular}

Lemma 1 Let $E_{t}^{i}$ be a certain equivalent class in a group $G_{i}$ and $\mathbb{E}_{t}^{i}$ be a set of other equivalent classes in $G_{i}$, except $E_{t}^{i}$. If the sum of none-counterfeit records in $\mathbb{E}_{t}^{i}$ for each sensitive value is more than or equal to the number of counterfeit records in $E_{t}^{i}$, then adversaries cannot identify counterfeit records in $E_{t}^{i}$

Proof Let $o_{t}^{i}(s)$ be the sum of none-counterfeit records for a certain sensitive value $s$ in $\mathbb{E}_{t}^{i}, u_{t}^{i}(s)$ be a sum of counterfeit records for $s$ in $\mathbb{E}_{t}^{i}$, and $p_{t}^{i}(s)$ be the number of counterfeit records for $s$ in $E_{t}^{i}$. Assume that at least one counterfeit record exists in $E_{t}^{i}$ and that $o_{t}^{i}(s)$ is more than or equal to $p_{s}$. To ensure that at least one counterfeit record exists in $E_{t}^{i}$, the following inequation should be satisfied by the pigeonhole principle.

$$
p_{t}^{i}(s)+u_{t}^{i}(s)>o_{t}^{i}(s)+u_{t}^{i}(s)
$$

It is contradictory to the assumption $p_{t}^{i}(s) \leq o_{t}^{i}(s)$.

The ID list should be organized for preserving privacy by Lemma 1. For example, Table 8 is a well-grouped catalog. Counterfeit Diabetes patients can be located in both class 1 and class 2 , and thus, adversaries cannot be sure where counterfeit records are located.

Table 5 is the 4 -anonymous and 0.02 -ceiled version of Table 1, and Table 8 shows the catalog of counterfeit records in Table 5. If Table 1 is generalized to satsify 0.02 -ceiling, 4-anonymity cannot be satisfied as shown in Table 6, because there are only three records in class 1 . Therefore, one counterfeit record is inserted to satisfy 4anonymity, as seen in Table 5 . The catalog shows that there is one counterfeit Diabetes patient record in a equivalent group of class 1 or class 2 . If data recipients require a more precise dataset without considering data truthfulness, they can use the anonymized dataset in Table 5 as it is. If the data recipients require a truthful dataset, they can handle the dataset as Table 9 using Table 8. The data recipients must remove the counterfeit record to obtain the truthful dataset. Although they cannot know exactly which equivalent class the counterfeit record belongs to, they can remove the counterfeit record using Table 8 . To remove the counterfeit record, the one Diabetes record in each equivalent class should be suppressed. After that, the recipients can remove one of the suppressed records because one of the suppressed records is a real record, and one is a counterfeit record. In [15], counterfeit records are inserted to protect the privacy only and there are no other methods to preserve the utility. On the other hand, in the

Table 8 Catalog of counterfeit records for Table 5

\begin{tabular}{lll}
\hline IDList & Disease & Count \\
\hline 1,2 & Diabetes & 1
\end{tabular}


Table 9 Truthful version of Table 5

\begin{tabular}{lllll}
\hline ClassID & Age & Sex & Zipcode & Disease \\
\hline 1 & {$[35-37]$} & F & {$[22071-23061]$} & Pneumonia \\
1 & {$[35-37]$} & F & {$[22071-23061]$} & Diabetes \\
1 & {$[35-37]$} & F & {$[22071-23061]$} & Anemia \\
2 & {$[61-66]$} & M & {$[55099-55324]$} & Pneumonia \\
2 & {$[61-66]$} & M & {$[55099-55324]$} & Diabetes \\
2 & {$[61-66]$} & M & {$[55099-55324]$} & Pneumonia \\
$*$ & $*$ & $*$ & $*$ & Diabetes \\
\hline
\end{tabular}

proposed method, counterfeit records are inserted to protect the privacy and preserve the utility. Furthermore, data utility can be preserved through the catalog.

\section{Quality metric}

In this paper, we adopted two anonymization methods, generalization and insertion of counterfeit records. Therefore, the data utility is determined by the information loss (caused by generalization) and the data truthfulness (caused by insertion). As we mentioned, existing metrics such as DM, LM are not sufficient for measuring quality of the counterfeit records. Because they measure information loss only through quasi-identifier transformation, counterfeit records and catalog cannot be considered in these metrics. For example, for measuring the utility by $\mathrm{LM}$, the most efficient anonymization method is adding $k-1$ counterfeit records to all original records; then LM is always zero. Therefore we will measure utility using RCE. RCE measures utility by using the difference between anonymized data and original data based on the probability density function. For instance, Fig. 2 shows the probability density function of the second record (Alice) for Age attribute in Table 1. Next, Fig. 3 shows the probability density function of the same record in Table 5 . Then, the information loss measured by RCE for Age attribute is the difference between Figs. 2 and 3. To calculate RCE of a record, the probability density function is generated for all attributes. The definition of RCE is as follows.

$$
R C E=\sum_{\forall t \in T} \int_{x \in D S}\left(\tilde{\mathcal{G}}_{t}(x)-\mathcal{G}_{t}(x)\right)^{2} \mathrm{~d} x
$$

where $D S$ is the dimensional space (including all the QI and sensitive attribute) of the record, $\tilde{\mathcal{G}}_{t}$ is the probability density function of the original dataset, and $\mathcal{G}_{t}$ is the probability density function of the anonymized dataset. In the proposed method, the probability density is changed owing to the catalog. In Table 5, there are four Diabetes patients in class 1 and class 2, and thus, the probability that the record is counterfeit is $25 \%$. Therefore, the probability density is reduced to $3 / 4$, as in Fig. 4 . We measure RCE through the difference between Figs. 2 and 4 .

\section{Implementation of anonymization algorithm}

In this section, we present the implementation of the anonymization algorithm using the proposed method. In our methods, we traverse the hierarchical lattice in a bottom-up manner and use heuristics for pruning the node. In addition, we propose a grouping algorithm for catalog publication. The proposed anonymization algorithm applies the full-domain generalization algorithm [16]. The full-domain generalization algorithm finds a solution in the hierarchical lattice that describes all the possible generalization cases as nodes. Many heuristic techniques have been proposed to reduce the search space. Traditional heuristic optimization methods for the algorithm focus on pruning the lattice using the monotonicity of $k$-anonymity [16, 17]. If a node satisfies the $k$-anonymity property, then all the parent nodes linked to that node satisfy $k$-anonymity. On the other hand, if

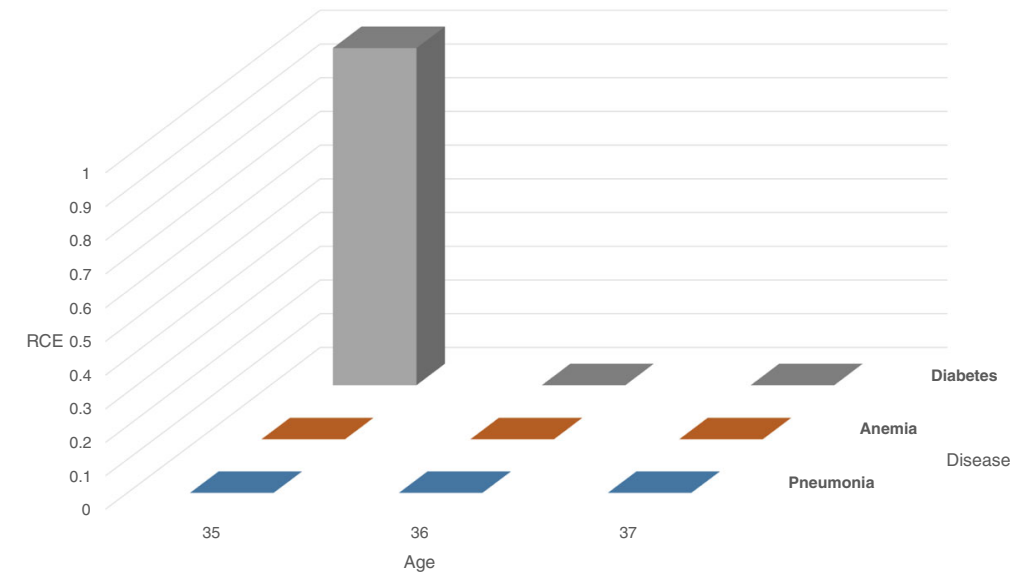

Fig. 2 Probability density function of original record for Age attribute 


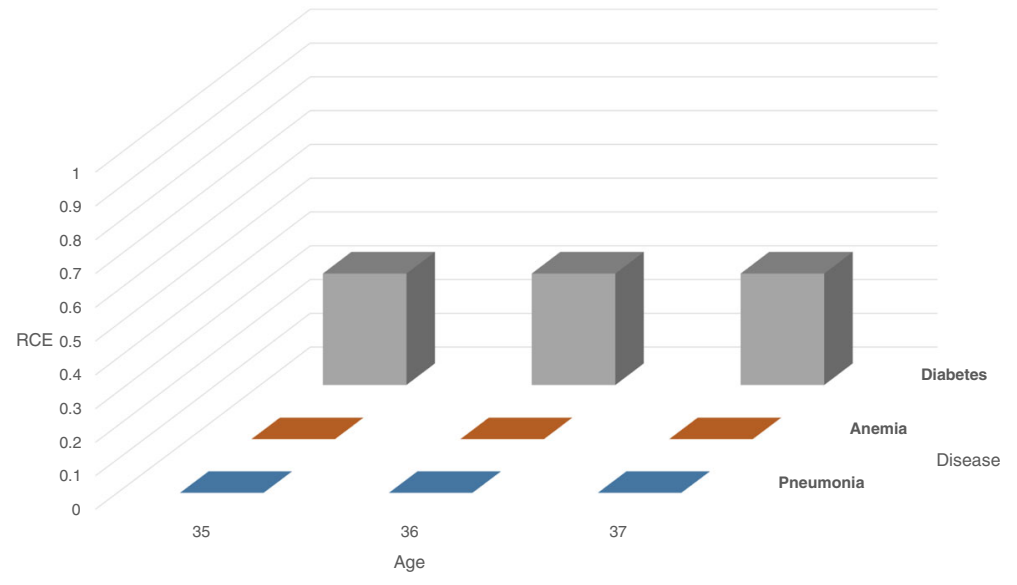

Fig. 3 Probability density function of generalized record for Age attribute

a node does not satisfy the $k$-anonymity property, then all the child nodes linked to that node do not satisfy $k$-anonymity. When using the proposed method, every node satisfies $k$-anonymity, and thus, the lattice cannot be pruned by the monotonicity of $k$-anonymity. Instead, we can prune the lattice by the monotonicity of $h$-ceiling. If a node does not satisfy $h$-ceiling, then all the parent nodes linked to the node cannot satisfy $h$-ceiling, and vice versa. In the proposed method, determining an appropriate value of $h$ is important for improving data utility. In our algorithm, if the value of $h$ is not determined, the algorithm searches every node in the lattice and finds an optimal value of $h$ based on RCE.

Algorithm 1 shows the anonymization algorithm. The algorithm begins with the creation of the hierarchical lattice. Pruning the lattice using monotonicity, we only create a part of the lattice, wherein the generalization boundary of a node is less than $h$ (line 1 ), and search all nodes (lines 3-13). When records are generalized, the number of records in a certain equivalent class can be less than $k$. Then, we add counterfeit records to the equivalent class and store the number of the counterfeit records in TempC (lines 7-13). After generalizing the records based on the generalization rule of the node, TempC is grouped (line 14). The Grouping function is described in Section 4.2. After grouping, the algorithm calculates data utility. If the catalog is null, the result is also null(line 15). In the completion phase, compare the data utility of each node and choose the node that has maximum data utility (lines 16-19).

For grouping a catalog with privacy concerns, we use the heuristic grouping method. Algorithm 2 is the pseudocode of the grouping algorithm. First, we create a list of the set $S_{e}$ about equivalent classes that have aggregated data in $\hat{T}^{*}$ and counterfeit records in TempC by sensitive information (line 1). For safe grouping, all equivalent

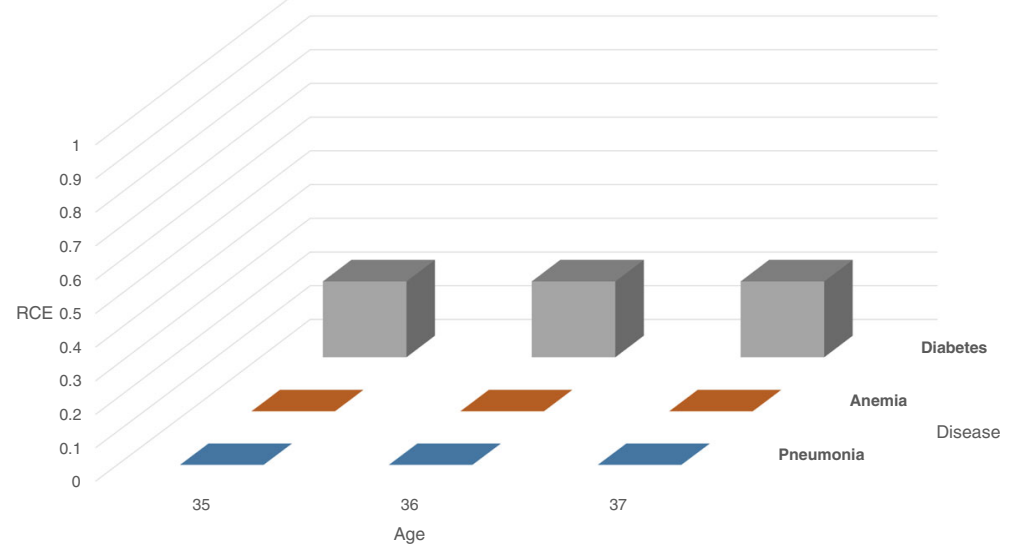

Fig. 4 Probability density function of generalized record for Age attribute with catalog 

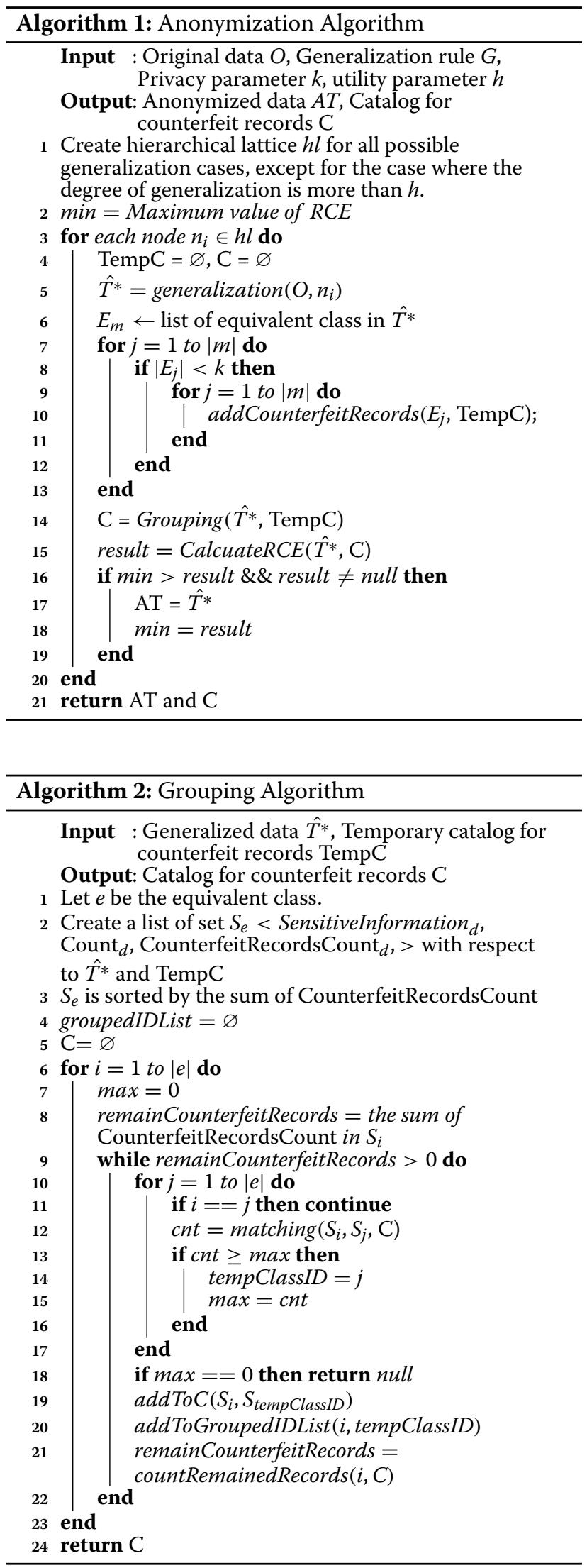

classes should find other equivalent classes that have sufficient none counterfeit records to conceal the counterfeit records by Lemma 1 . It is harder to find other equivalent classes when the equivalent class has more counterfeit records. Thus, the list $S_{e}$ is sorted since the equivalent class that is hard to group takes priority (line 3). After sorting, the algorithm finds equivalent classes grouped into the catalog in descending order (lines 6-23). In this phase, we count matched records to find the most appropriate equivalent class. The matching function returns the matched count of the counterfeit records of $S_{i}$ and the none counterfeit records of $S_{j}$. If $S_{i}$ or $S_{j}$ is already grouped, then the counterfeit records of $S_{i}$ can match the none counterfeit records of another set $S$ in the group (line 12). matching function returns null for all equivalent classes while remainCounterfeitRecords is over 0 , the equivalent class $S_{i}$ cannot be protected by grouping; hence, the algorithm returns null (line 18). If the most appropriate equivalent class is found, the algorithm groups the equivalent classes and counts the unconcealed records and puts that records into remainCounterfeitRecords (lines 18-21). If the counterfeit records cannot be concealed at a time, the process is repeated.

\section{Results and discussions}

In this section, we present the experimental evaluation of the proposed method. For the evaluation, we use the Adult dataset from the UCI Machine Learning Repository [18], which is a de facto standard dataset for measuring the quality of anonymization algorithms. The Adult dataset has 15 attributes and 32,561 rows. In our experiments, we use eight features, seven quasi-identifiers, and one sensitive attribute for all rows. We used three metrics to assess loss of information: (1) LM, (2) RCE, and (3) query error rate.

In addition, we evaluate the effectiveness of the proposed method in a real world analysis through the NPS dataset from HIRA (Health Insurance Review and Assessment service in Korea) [19]. The NPS (National Patients Sample) dataset consists of EHRs of 3\% sampled Korean people in 2011. We analyze 1,375,900 records with 6 attributes: Age, Sex, Length of stay in hospital, Location, Surgery status, and Disease. We consider the first five attributes to be quasi identifiers and the disease attribute to be a sensitive attribute. In the experiments, in addition to reporting results for the $k$-anonymity with $h$-ceiling method presented in this paper, we also report results for the existing $k$-anonymity algorithm in [16].

\section{LM}

LM is a general metric for measuring information loss caused by generalization. Figures 5 and 6 show the variation in LM with respect to the privacy parameter $k$ and 


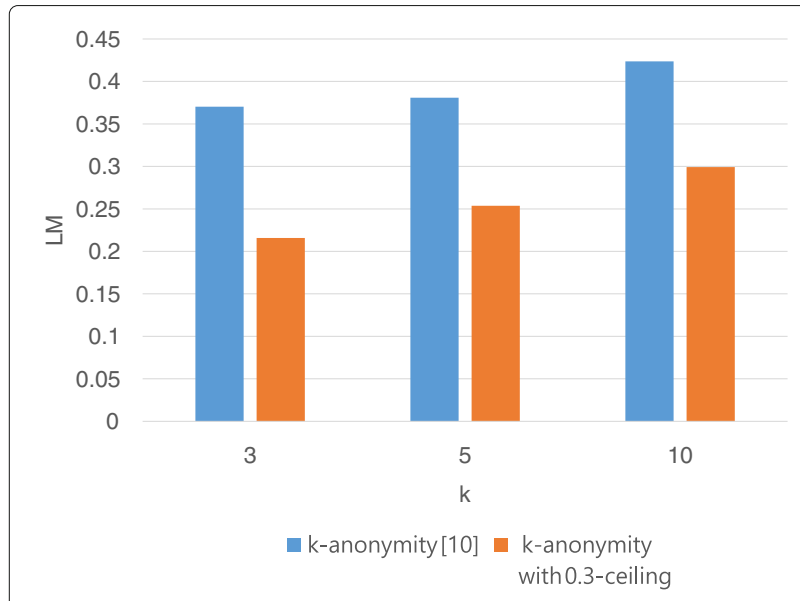

Fig. $5 \mathrm{LM}$ variation with $\mathrm{k}$ (The lower the bar in the graph, the better are the results)

the utility parameter $h$, respectively. In Fig. 5 , we compare the $h$-ceiled dataset and the $k$-anonymous datasets when $h$ is fixed to 0.3 while the value of $k$ varies. The $k$ anonymous datasets have at least $0.37 \mathrm{LM}$ when $k$ is 3 ; $\mathrm{LM}$ increases to more than 0.4 when $k$ is 10 . This is because the data are more generalized to satisfy stricter privacy constraints; that is, LM is proportional to $k$. However, the $h$-ceiled dataset has at most $0.3 \mathrm{LM}$ when $k$ is 10 because we constrain the generalization degree by $h$-ceiling. The counterfeit records are inserted with 125, 132, and 103 as the value of $k$ increases from 3 to 10. Figure 6 illustrates the LM when $k$ is fixed to 5 and $h$ varies. The counterfeit records are inserted with 138,132 , and 38 as the value of $h$ increases from 0.25 to 0.35 . LM increases with $h$. This is because counterfeit records that can decrease LM are inserted to minimize RCE according to the anonymization algorithm. Therefore, LM increases to reduce RCE.

\section{RCE}

In our anonymization method, RCE measures information loss instead of LM, because LM cannot reflect the

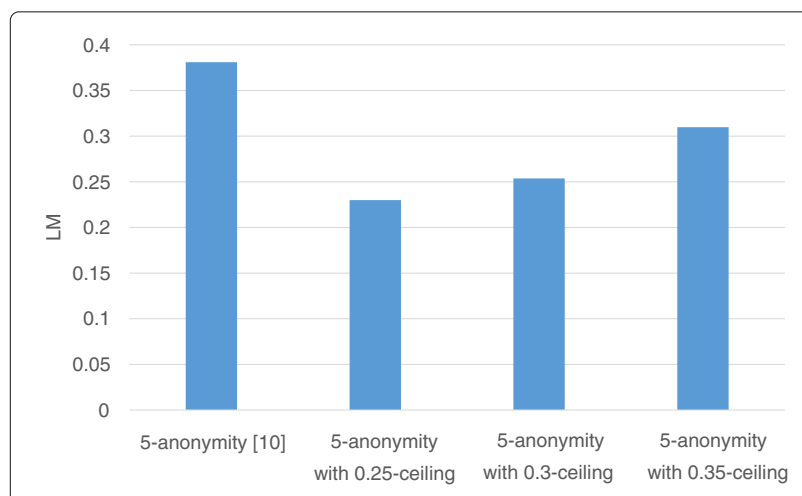

Fig. 6 LM variation with $h$

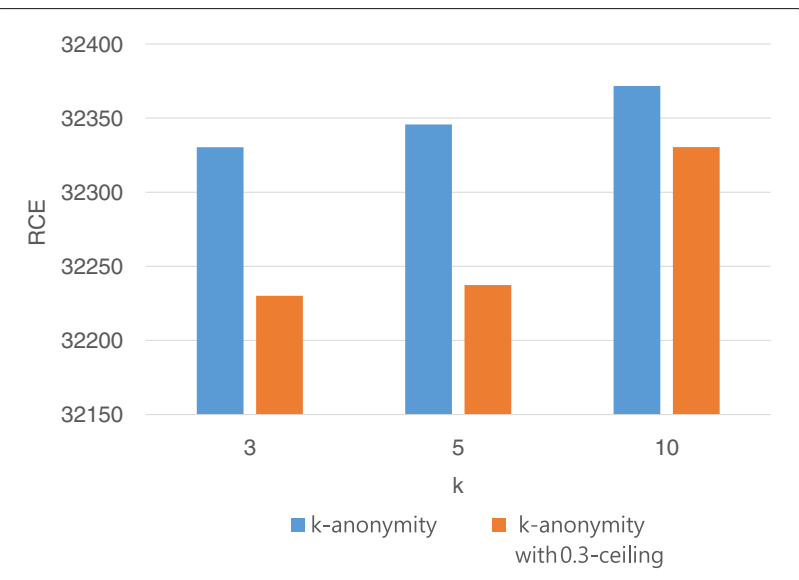

Fig. 7 RCE variation with $k$ (The lower the bar in the graph, the better are the results)

distortion of counterfeit records. Figures 7 and 8 show the variation in RCE with respect to the privacy parameter $k$ and the utility parameter $h$, respectively. Figure 7 shows the comparison between the $h$-ceiled dataset and $k$-anonymous dataset when $h$ is fixed to 0.3 and the value of $k$ varies. In Fig. 7, $h$ is fixed to 0.3 and the value of $k$ is varied; the result is compared with $\mathrm{k}$-anonymous data. RCE increases quite a bit when the value of $k$ is 10 because a lot of counterfeit records are created to satisfy privacy constraints. Nevertheless, the loss of results in the $h$-ceiled dataset is less than that of results in k-anonymous data. Figure 8 illustrates the variation in RCE with the value of $h$. As the parameter $h$ decreases from 0.35 to 0.25 , RCE increases. This is because a lot of counterfeit records were created owing to the strict constraint of the generalization degree.

\section{Query error rate}

We show the result of range queries to verify the effectiveness of the proposed method. We fix the value of $k$ as 5 and the value of $h$ as 0.3 in these experiments. Figure 9 shows

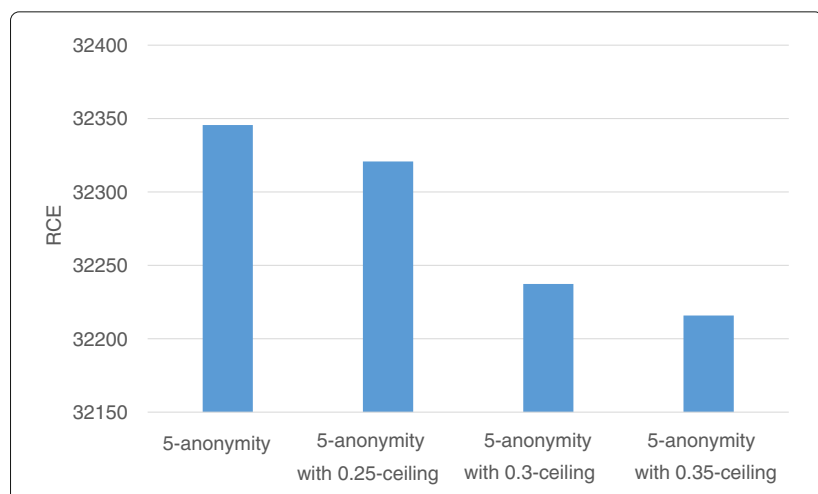

Fig. 8 RCE variation with $h$ 


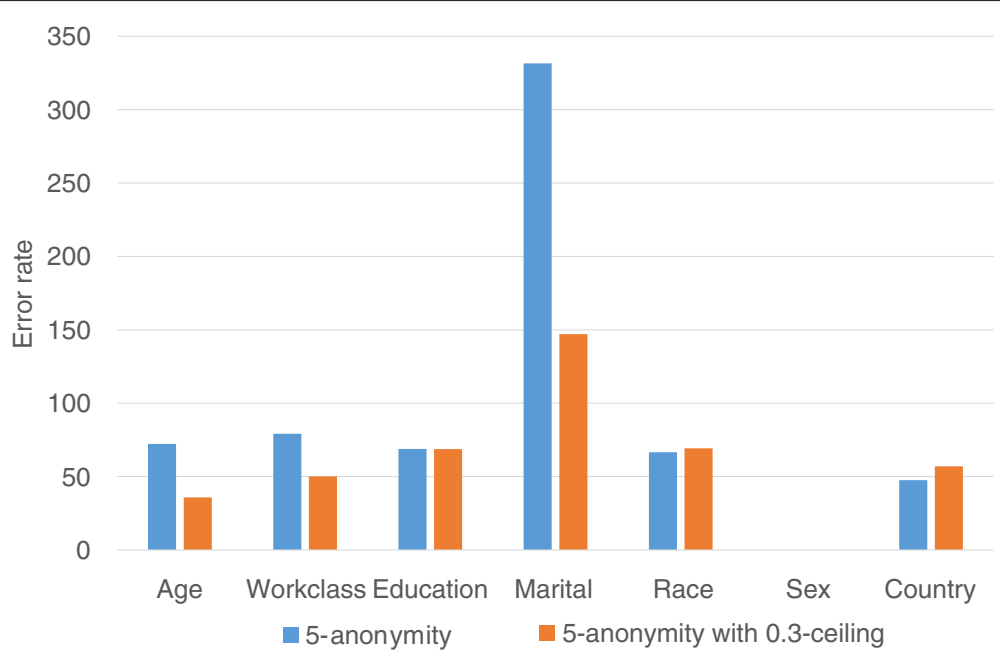

Fig. 9 Query error rate

that the error rate of the query result for each attribute. We performed an aggregation query such as SELECT COUNT(*) FROM Adultdataset WHERE Occupation = 'Sales' GROUP BY Age on both the original dataset and the anonymized dataset, and then compared the results. In Adult dataset, the marital status is skewed, and thus, the error rate of the query result on marital status is almost $300 \%$. However, when the proposed method is applied, the error rate can be reduced by half. Further, the error rate on the other attribute for the $h$-ceiled data is also less or nearly equal to the $k$-anonymous data. The error rate on attribute $\operatorname{Sex}$ is 0 because this attribute is not generalized. Only two values, man and woman, are in this attribute, and thus, the height of the taxonomy tree is 2. This means that if the attribute is generalized, the generalization degree becomes 1 although it is generalized by only one level. In Fig. 10, we show the variation in query error rate with the number of

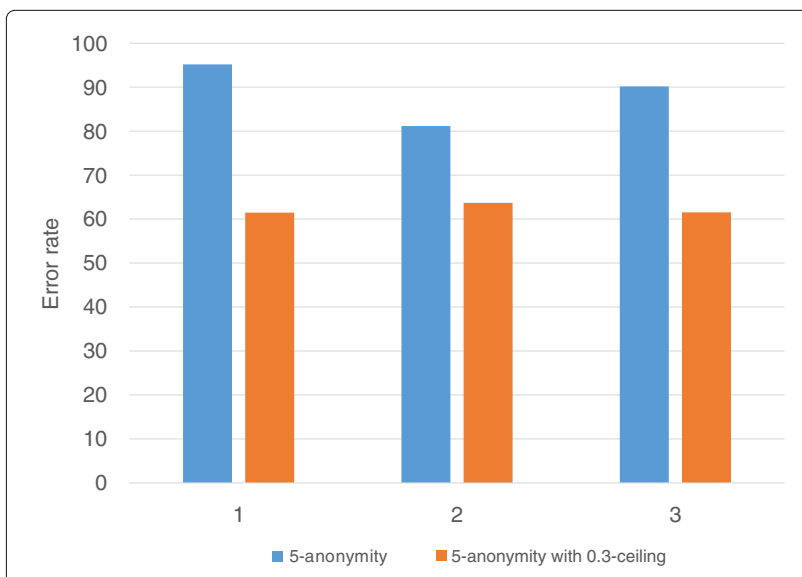

Fig. 10 Query error rate variation with number of attributes attributes. The error rate on the $h$-ceiled dataset is less than the $k$-anonymous data, regardless of the number of attributes.

\section{Real world analysis}

We present a real-world analysis to illustrate the usefulness of the proposed method. We experimented using the statistical analysis queries used in HIRA [19]. The dataset is anonymized in two ways: (1) $k$-anonymization with $k=10$, and (2) using the proposed method with $k=10$ and $h=0.2$. We further compare the results of the anonymized data with those of the original data by using aggregation queries. The queries are described as follows.

- $Q_{1}$ : SELECT FLOOR(Age/5)*5 AS AgeGroup, COUNT(") AS No._of_patients FROM NPS dataset WHERE Sex $=$ 'M' and Surgery status $=$ 'N' and Disease $=$ 'stroke' GROUP BY FLOOR(Age/5)*5

- $Q_{2}$ : SELECT FLOOR(Age/5)*5 AS AgeGroup, COUNT (*) AS No._of_patients FROM NPS dataset WHERE Sex $=$ 'F' and Surgery status $=$ 'N' and Disease $=$ 'stroke' GROUP BY FLOOR(Age/5)*5

- $Q_{3}$ : SELECT FLOOR(Age/5)*5 AS AgeGroup, AVG(Length of stay in hospital) AS Average_length_of_stay_in_hospital FROM NPS dataset WHERE Sex = 'M' and Surgery status $=$ 'N' and Disease $=$ 'stroke' GROUP BY FLOOR $($ Age $/ 5) * 5$

- Q4: SELECT FLOOR(Age/5)*5 AS AgeGroup, AVG(Length of stay in hospital) AS Average_length_of_stay_in_hospital FROM NPS dataset WHERE Sex = 'F' and Surgery status $=$ ' $\mathrm{N}$ ' and Disease $=$ 'stroke' GROUP BY FLOOR(Age/5)*5 


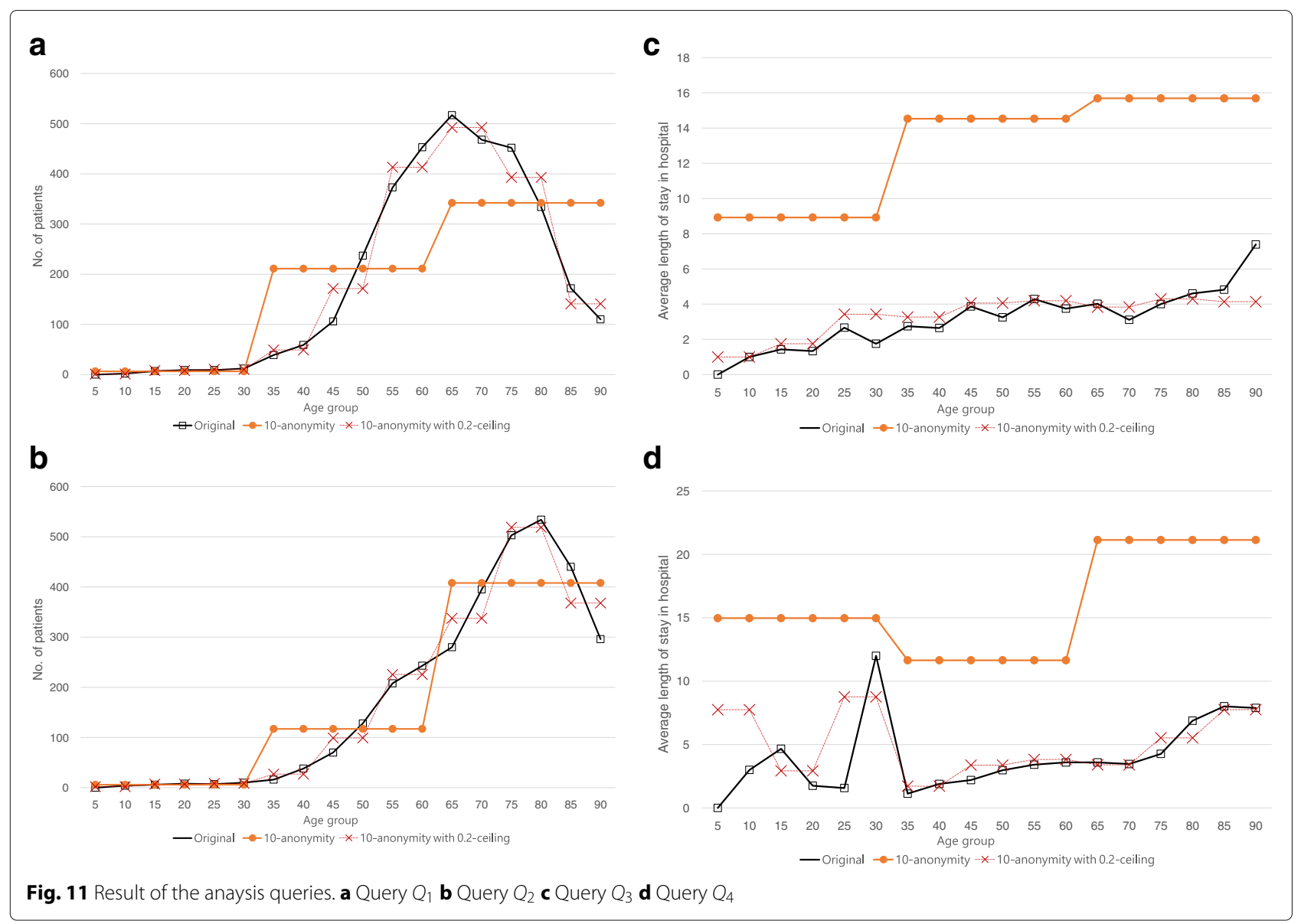

$Q_{1}$ and $Q_{2}$ represent the number of stroke patients for each age group $(0-4,5-9, \ldots, 86-90) . Q_{3}$ and $Q_{4}$ represent the average length of stay in hospital. Figure 11 shows the results of the analysis queries. In Fig. 11-(a) and (b), the $\mathrm{x}$-axis represents the age group (which corresponds to the first projection column of $Q_{1}$ and $Q_{2}$ ) and the y-axis represents the number of stroke patients (which corresponds to the second projection column of $Q_{1}$ and $Q_{2}$ ). In Fig. 11-(c) and (d), the $\mathrm{x}$-axis represents the age group (which corresponds to the first projection column of $Q_{3}$ and $Q_{4}$ ) and the $y$-axis represents the average length of stay in hospital for stroke patients (which corresponds to the second projection column of $Q_{3}$ and $Q_{4}$ ). In each figure, the result of the $h$-ceiled data is more similar to the original data than that of the $k$-anonymous data. Especially, the proposed method shows better performance in Fig. 11-(c) and (d). Because the average length of stay in hospital is a generalized value, the errors increase as the degree of generalization increases.

\section{Conclusions}

In this paper, we proposed a utility-preserving anonymization method for organizing $h$-ceiled and $k$-anonymous data using two main ideas: counterfeit records, and catalog. Furthermore, we devised an anonymization algorithm with a grouping algorithm and a precise measuring metric of data utility by RCE. Through our experimental results, we demonstrated that $h$-ceiling can prevent the over-generalization and the catalog can preserve data truthfulness of an anonymized dataset.

As possible future works, we can design new anonymization methods that can satisfy $h$-ceiling with other privacy preserving models such as $l$-diversity, $t$ closeness, or $\mathrm{MS}\left(k, \theta^{*}\right)$-anonymity [20-22]. In this paper, we considered only the full-domain generalization which is the most widely used anonymization methodology especially in health/medical domains $[16,17,23,24]$. As future work, we need extend the proposed method to other types of generalization mechanisms such as clustering and Mondrian [25, 26].

\section{Abbreviations}

DM: Discernibility metric; EHR: Electronic health record; HIPAA: US Health Insurance portability and accountability act; HIRA: Health insurance review and assessment service in Korea; LM: Loss metric; NPS: National patients sample; PPDP: Privacy preserving data publishing; Pll: Personally identifiable information; RCE: ReConstruction error 


\section{Acknowledgements}

This work was supported by Institute for Information \& communications Technology Promotion(IITP) grant funded by the Korea government(MSIP) (No.R0190-15-2019, Development of personal information protection technology using unidentifiability technique on big data environment).

\section{Availability of data and materials}

The datasets generated and analysed during the current study are available in the UCI Machine Learning Repository [18] and the HIRA repository [19].

\section{Authors' contributions}

$\mathrm{HL}$ designed the study, performed analysis, and drafted the manuscript. HL and SK conducted the evaluation. JWK and YDC reviewed the manuscript. YDC contributed to the discussion, assisted with and supervised the design of the study. All authors read and approved the final manuscript.

\section{Ethics approval and consent to participate}

Not applicable.

\section{Consent for publication}

Not applicable.

\section{Competing interests}

The authors declare that they have no competing interests.

\section{Publisher's Note}

Springer Nature remains neutral with regard to jurisdictional claims in published maps and institutional affiliations.

\section{Author details}

${ }^{1}$ Department of Computer Science and Engineering, Korea University, 145 Anam-ro, Seongbuk-gu, 02841, Seoul, Republic of Korea. ${ }^{2}$ Department of IT Convegence, Korea University, Seoul 145 Anam-ro, Seongbuk-gu, 02841, Republic of Korea. ${ }^{3}$ Department of Media Software, Sangmyung University, Seoul 20-Gil, Hongji-dong, Seongbuk-gu, 03016, Republic of Korea.

Received: 6 April 2017 Accepted: 28 June 2017

Published online: 11 July 2017

\section{References}

1. Holzinger A, Dehmer M, Jurisica I. Knowledge discovery and interactive data mining in bioinformatics-state-of-the-art, future challenges and research directions. BMC Bioinforma. 2014;15(6):1.

2. Holzinger A, Jurisica I. Knowledge discovery and data mining in biomedical informatics: The future is in integrative, interactive machine learning solutions. In: Interactive Knowledge Discovery and Data Mining in Biomedical Informatics. Berlin: Springer; 2014. p. 1-18.

3. Malle B, Kieseberg P, Schrittwieser S, Holzinger A. Privacy aware machine learning and the right to be forgotten. ERCIM News. 2016;107(10):22-3.

4. Sweeney L. K-anonymity: A model for protecting privacy. Int J Uncertain Fuzziness Knowledge-Based Syst. 2002;10(05):557-70.

5. Family Educational Rights and Privacy Act. 2015. Available at https://ed. gov/policy/gen/guid/fpco/ferpa.

6. Guidelines for De-identification of Personal Data. 2016. Available at http:// privacy.go.kr.

7. Kieseberg $P$, Malle B, Frühwirt P, Weippl E, Holzinger A. A tamper-proof audit and control system for the doctor in the loop. Brain Inform. 2016;3(4):269-79.

8. Sweeney L. Achieving k-anonymity privacy protection using generalization and suppression. Int J Uncertain Fuzziness Knowledge-Based Syst. 2002;10(05):571-88.

9. Nergiz ME, Gök MZ. Hybrid k-anonymity. Comput Secur. 2014;44:51-63.

10. Prasser F, Kohlmayer F, Kuhn KA. Efficient and effective pruning strategies for health data de-identification. BMC Med Inform Decis Making. 2016;16(1):49.

11. Fung BC, Wang K, Fu AW-C, Philip SY. Introduction to Privacy-preserving Data Publishing: Concepts and Techniques. Boca Raton: CRC Press; 2010.

12. Bayardo RJ, Agrawal R. Data privacy through optimal k-anonymization. In: Proceedings of the 21st International Conference on Data Engineering. Tokyo: IEEE Computer Society; 2005. p. 217-28.
13. lyengar VS. Transforming data to satisfy privacy constraints. In: Proceedings of the Eighth ACM SIGKDD International Conference on Knowledge Discovery and Data Mining. Edmonton: ACM; 2002. p. 279-88.

14. Xiao X, Tao Y. Anatomy: Simple and effective privacy preservation. In: Proceedings of the 32 nd International Conference on Very Large Data Bases. Seoul: VLDB Endowment; 2006. p. 139-50.

15. Xiao X, Tao Y. M-invariance: Towards privacy preserving re-publication of dynamic datasets. In: Proceedings of the 2007 ACM SIGMOD International Conference on Management of Data. Beijing: ACM; 2007. p. 689-700.

16. Kohlmayer F, Prasser F, Eckert C, Kemper A, Kuhn KA. Flash: Efficient, stable and optimal k-anonymity. In: Proceedings of the 2012 ASE/IEEE International Conference on Social Computing and 2012 ASE/IEEE International Conference on Privacy, Security, Risk and Trust. Amsterdam: IEEE Computer Society; 2012. p. 708-17.

17. El Emam K, Dankar FK, Issa R, Jonker E, Amyot D, Cogo E, Corriveau JP, Walker M, Chowdhury S, Vaillancourt R, et al. A globally optimal k-anonymity method for the de-identification of health data. J Am Med Inform Assoc. 2009;16(5):670-82.

18. UCI Repository of Machine Learning Databases. 2013. Available at http:// archive.ics.uci.edu/ml.

19. Health Insurance Review and Assessment Service in Korea. 2012. Available at http://opendata.hira.or.kr.

20. Machanavajjhala A, Kifer D, Gehrke J, Venkitasubramaniam M. I-diversity: Privacy beyond k-anonymity. ACM Trans Knowl Discov Data (TKDD). 2007;1 (1):3.

21. Li N, Li T, Venkatasubramanian S.t-closeness: Privacy beyond k-anonymity and I-diversity. In: Proceedings of the 21st International Conference on Data Engineering. Istanbul: IEEE Computer Society; 2007. p. 106-15.

22. Lin WY, Yang DC, Wang JT. Privacy preserving data anonymization of spontaneous ade reporting system dataset. BMC Med Inform Decis Making. 2016;16(1):58

23. Kohlmayer F, Prasser F, Eckert C, Kemper A, Kuhn KA. Highly efficient optimal k-anonymity for biomedical datasets. In: Computer-Based Medical Systems (CBMS), 2012 25th International Symposium On. Rome: IEEE; 2012. p. 1-6.

24. Kohlmayer F, Prasser F, Kuhn KA. The cost of quality: Implementing generalization and suppression for anonymizing biomedical data with minimal information loss. J Biomed Inform. 2015;58:37-48.

25. Byun JW, Kamra A, Bertino E, Li N. Efficient k-anonymization using clustering techniques. In: International Conference on Database Systems for Advanced Applications. Berlin: Springer; 2007. p. 188-200.

26. LeFevre K, DeWitt DJ, Ramakrishnan R. Mondrian multidimensional k-anonymity. In: Data Engineering, 2006. ICDE'06. Proceedings of the 22nd International Conference On. Atlanta: IEEE; 2006. p. 25-5.

\section{Submit your next manuscript to BioMed Central and we will help you at every step:}

- We accept pre-submission inquiries

- Our selector tool helps you to find the most relevant journal

- We provide round the clock customer support

- Convenient online submission

- Thorough peer review

- Inclusion in PubMed and all major indexing services

- Maximum visibility for your research

Submit your manuscript at www.biomedcentral.com/submit
C Biomed Central 